\title{
Cholecystitis and Synchronous Liver Abscess: Percutaneous Treatment Results
}

\section{Dos Santos Gabriel ${ }^{1}$, Voliovici Eduardo ${ }^{2}$ and Olivera Pertusso Eduardo ${ }^{3 *}$}

${ }^{1}$ Ex Resident at Surgery Department, Medical School, Universidad de la República, Uruguay

${ }^{2}$ Associate Professor at Surgery Department A, Medical School, Universidad de la República, Uruguay

${ }^{3}$ Added Professor at Surgery Department, Anatomy Director Professor, Medical School, Universidad de la República, Uruguay

*Corresponding Author: Pertusso Eduardo, Added Professor at Surgery Department, Anatomy Director Professor, Medical School, Universidad de la República, Uruguay.

Received: September 30, 2019; Published: October 15, 2019

DOI: $10.31080 /$ ASGIS.2019.02.0089

\begin{abstract}
Introduction: Liver abscesses associated to acute cholecystitis are a rare but yet a serious complication, and there are only a few cases reported. The treatment is based on the percutaneous drainage guided by images and deferred Cholecystectomy, a technique associated to low morbidity and mortality.

The objective is to communicate the results based on our experience with the percutaneous treatment of this complication.

Materials and Methods: Retrospective analysis of 20 patients, from July 2012 to September 2017.

Results: 20 patients were studied, $60 \%$ men, $40 \%$ women, average age of 72 . They presented fever $55 \%$ and acute abdominal pain $60 \%$. The average leukocytosis was 17000 white blood cells. $30 \%$ of the patients were diabetic and $25 \%$ of them had malignant neoplasms. $95 \%$ were diagnosed by CT Scan. $85 \%$ showed gallbladder lithiasis. $80 \%$ showed a unique abscess. Positive cultures were found in $65 \%$ of the patients, and the most frequent germ was Klebsiella Pneumoniae. One patient (5\%) showed a hematoma as a complication. The drainage was effective in $100 \%$ of the patients and they all had a good evolution except for one patient who passed of severe sepsis. No patient required urgent surgery; coordinated Cholecystectomy was performed to $20 \%$.

Conclusion: Liver abscesses associated to acute cholecystitis are rare and severe.

Diagnosis is based on clinical suspicion and image confirmation.

The barely invasive treatment, percutaneous, guided by images is the treatment of choice. It is a treatment that consists of the drainage of the abscess and/or Cholecystectomy. It is a very effective treatment with a very low rate of morbidity and mortality, and in most cases definitive.

Keywords: Cholecystitis; Percutaneous
\end{abstract}

\section{Introduction}

Although Acute Cholecystitis it's a high prevalence entity, its association with perivascular liver abscess it's not frequent and this complication represents a rate of less than $1 \%$ [1-3].

The most frequent cause of liver abscesses secondary to an acute Cholecystitis is the intrahepatic perforation of the gallblad$\operatorname{der}[4]$.

There are only a few published cases about liver abscesses secondary to intrahepatic perforation of the gallbladder [3-6]. The treatment in these cases with severe inflammatory elements is Cholecystectomy and the surgical or percutaneous drainage of the abscess [3].
The objective of this work is to communicate the results of our experience with percutaneous drainage treatment in this rare complication of an acute Cholecystitis.

\section{Materials and Methods}

A retrospective study was made with 20 patients treated with the percutaneous technique, from July 2012 to September 2017 at different medical centers.

- $\quad$ The following parameters were analyzed: Age, gender, comorbidities, sepsis, bacteriology, the presence of gallbladder lithiasis, effectiveness of the percutaneous drainage, complications, Cholecystectomy in the evolution, and mortality in 30 days. 


\section{Results}

Twenty patients were treated, 12 men $(60 \%)$ and 8 women (40\%) (Table 1), and the average age was 72 (48-95). There were 11 admitted with fever (55\%), while 12 (60\%) presented abdominal pain at right hypocondrium.



Table 1: Distribution by sex.

The average leukocytosis was 17000 white blood cells (609025600 WBC) (Table 2). Two patients presented severe sepsis (10\%), $30 \%$ of the patients were diabetic, $50 \%$ presented other medical history such as Hypertension, Respiratory infections, aortic stenosis, Ischemic Cardiopathy, and frequent urinary infections. Five patients (25\%) had malignant neoplasms. Nineteen patients (95\%) were diagnosed via CT Scan and only one patient was diagnosed with ultrasound as an exclusive method. Gallbladder lithiasis was diagnosed in $85 \%$ of the patients. Sixteen (80\%) patients presented a unique abscess and 4 patients $(20 \%)$ presented multiple ones, 2 to 4 liver abscesses. One patient presented an interhepatofrenic abscess and another present a left subhepatic abscess (Figure 1). About bacteriology, positive cultures were found in 13 patients (65\%), and the most frequent germ found was Klebsiella Pneumoniae (6 patients), followed by Escherichia Coli (4 patients). Other germs found were Enterococcus Faecalis, Serratia, S. Aureus, Pseudomona and Clostridium Perfringens. In 2 patients, the culture was positive for 3 germs at once (Table 3). All patients received antibiotics and they were adjusted according to the culture results. Percutaneous drainage was performed to all patients. 6 patients were treated with more than one drainage. The remaining 14 patients had the gallbladder and the abscess treated with the same drainage.

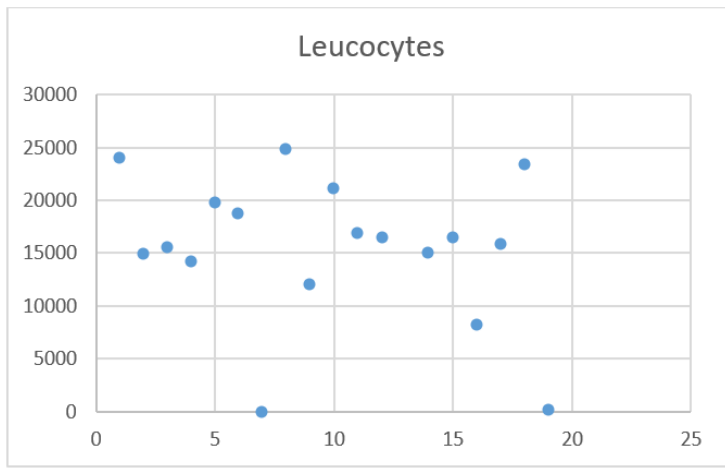

Table 2: Leukocytosis found at the time of consultation of the patients.

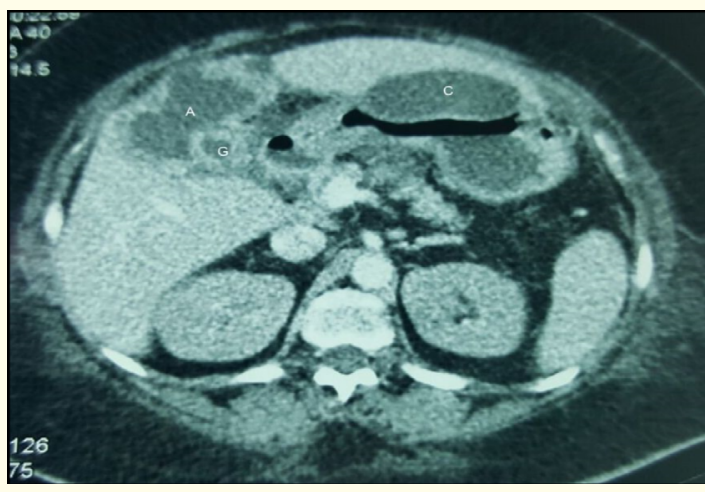

Figure 1: CT shows the gallbladder $(\mathrm{G})$, liver abscess $(\mathrm{H})$ and a left subhepatic collection (C).

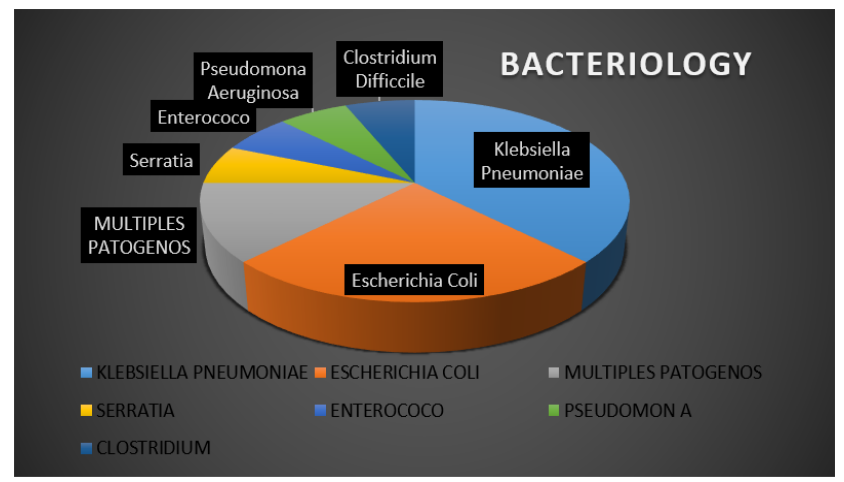

Table 3: Germs found in abscess cultures.

The mortality in 30 days was $5 \%$ (1 patient) who was admitted with severe sepsis and an abscess with gas, with a positive culture for Clostridium Perfringens.

Of all of our patients only one of them presented a complication, a haematoma that required readmittance and placing of a new percutaneous drainage.

The percutaneous drainage was effective in $100 \%$ of the patients, and none of them required an acute surgery procedure for its definitive resolution. All the patients had a good evolution except for one of them who passed early of severe sepsis.

In the evolution, coordinated Cholecystectomy was performed to $20 \%$ of the patients.

\section{Discussion}

Acute Cholecystitis is a frequent pathological entity but liver abscesses as a complication are rare [1-3,7].

The most frequent etiology of acute Cholecystitis is the obstructive or mechanical one secondary to gallbladder lithiasis in more than $95 \%$ of the cases. After the impact of a gallstone into the gallbladder bacinete or at the cystic duct a series of pathogenic events occur which include a gallbladder distension and inflammation of 
the gallbladder walls with an added bacterial infection. The distension and ischemia generated over the gallbladder wall lead to its perforation in the evolution if not treated properly $[2,8]$.

The perforation can occur either early or late after weeks of the beginning of the clinical picture [3], depending of factors such as bacterial infection, subacute inflammatory process with the formation of adhesions, use of corticosteroids, Diabetes, vascular disease, old age and female gender [9].

Different pathological clinical pictures are determined according to the site of perforation and the presence of an inflammatory process, which objective is to delimit the infectious focus $[3-5,7,9]$.

In 1934 Niemeier [4] proposes a classification of 3 types, which is then modified by Fletcher and Radvein [10] in 1951, describing 3 types of perforation: Type 1, acute perforation in the peritoneal cavity; Type 2, subacute perforation with formation of a pericholecystic abscess, and Type 3, chronic perforation with formation of a cholecystoenteric fistula. In 1987 Anderson, based on an analysis of cases with evidence of formation of cholecystobiliary fistulas, proposes a modification adding a fourth type of perforation, the cholecystobiliary fistula [11]. Intrahepatic perforation has not been defined as a particular type according to this classification and the different reports include them into type 3 (cholecystovisceral fistula) $[3,5,6]$.

The formation of a Liver abscess as a complication of an acute Cholecystitis is rare. Intrahepatic perforation of the gallbladder is also rare and it is usually present in particular anatomic forms of the gallbladder (intrahepatic gallbladder) [3,5,6,12-16].

There are only a few cases of liver abscesses secondary to an intrahepatic perforation of the gallbladder described in the bibliography $[3,5,6,10,12-21]$.

The precise mechanism of the formation of a liver abscess in the acute Cholecystitis remains speculative, and possible routes are posed such as a direct invasion of the liver parenchyma, a lymphatic subcapsular way, or a hematogenous form through the Portal Vein. This is particularly debatable in small and multiple abscesses located far from the gallbladder [22,23]. However, the perforation of the gallbladder to the liver determines the formation of an abscess mainly when an intrahepatic gallbladder exists $[3,5,6]$. The formation of a cholecystohepatic fistula is generated [5].

In many of the reported cases, many patients had a partial gallbladder or totally intrahepatic [3,5,6,12-21]. Therefore, the rupture of an intrahepatic gallbladder determines a liver located abscess without the formation of adhesions (plastron) or generalized peritonitis [3].
The pyogenic liver abscesses are a severe entity which also represents a dare to the clinical diagnosis. The classic signs which include fever, abdominal pain, painful hepatomegaly and jaundice are always present. Clinical manifestations vary from patients with fever and overall impact to formations that simulate liver tumors $[3,5,21,22]$.

However, the majority of symptoms are present in the acute Cholecystitis, reason why it becomes difficult to discriminate clinically among patients with intrahepatic perforation and those without it. The fast pain relief can be a consequence of the gallbladder perforation due to a decrease of the pression inside the gallbladder $[3,5,16]$

The abdominal ultrasound allows us to evaluate with great precision the liver and the biliary pole. The ultrasound signs are diverse and not specific to this complication. Besides the classic ultrasound signs of Cholecystitis and the ultrasound signs of a liver abscess, this technique lets us suspect an intrahepatic perforation indirectly $[3,5,13]$. An ultrasound sign named "the sign of the hole" is highly suggestive of Cholecystohepatic fistula [3,13,19] (Figure 2).

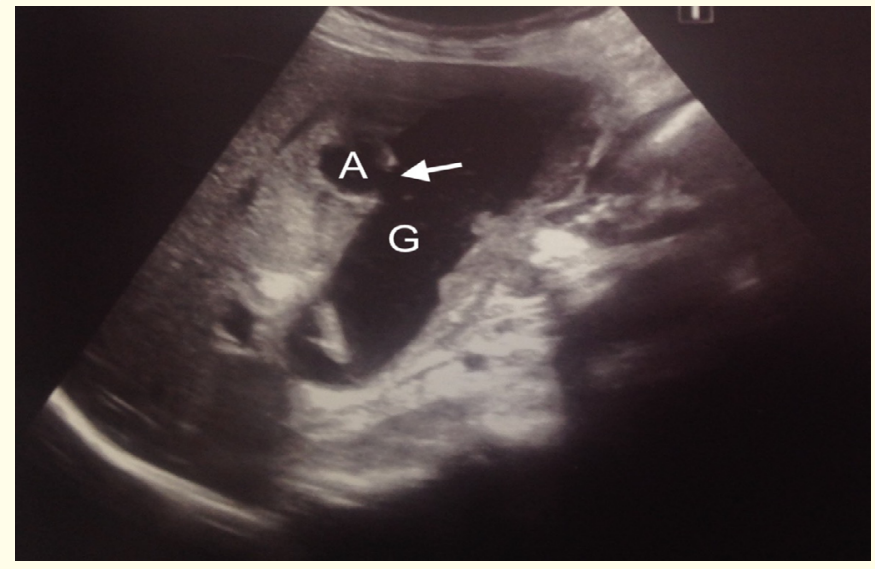

Figure 2: Ultrasound shows the sign of the hole (white arrow). Gallbladder (G), abscess (A).

The lack of visualization of the gallbladder in the presence of a liver abscess, a liver abscess in continuity with the gallbladder or the presence of gallstones inside the abscess, as well as the absence of peri vesicular collections are highly suggestive of an intrahepatic perforation with a communication with a cholecystohepatic fistula [3].

The abdominal ultrasound don't allows a clear diagnosis of the etiology of the abscess because sometimes the gallbladder is not visualized properly [3]. The CT Scan becomes important to the diagnosis of the intrahepatic perforation (Figure 3). Many tomographic signs have been described. They are direct signs of the presence 
of calculi outside the gallbladder and where the abscess is, or the identification of a partial gallbladder wall rupture $[3,13,19]$. The presence of an abscess next to the gallbladder or the presence of a thickened gallbladder wall are indirect signs. The identification of a communication hole between the gallbladder and the abscess is not always possible (sign of the hole) [3,13,19] (Figure 4, 5 and 6).

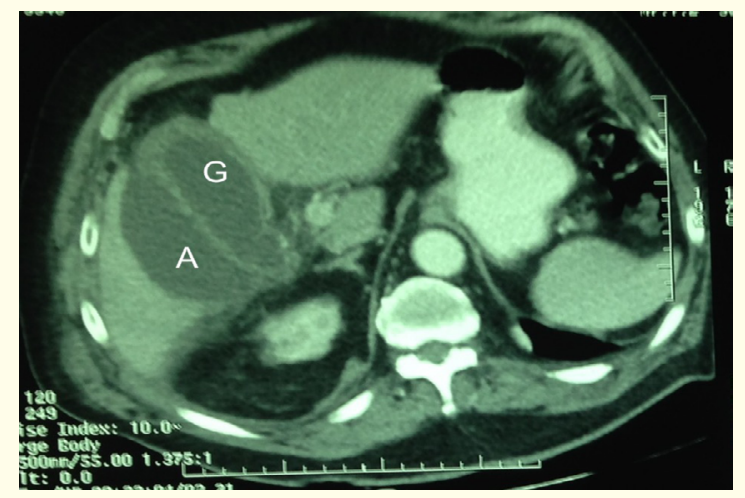

Figure 3: CT shows acute cholecystitis with a perivesicular liver abscess. Gallbladder (G) Liver abscess (A).

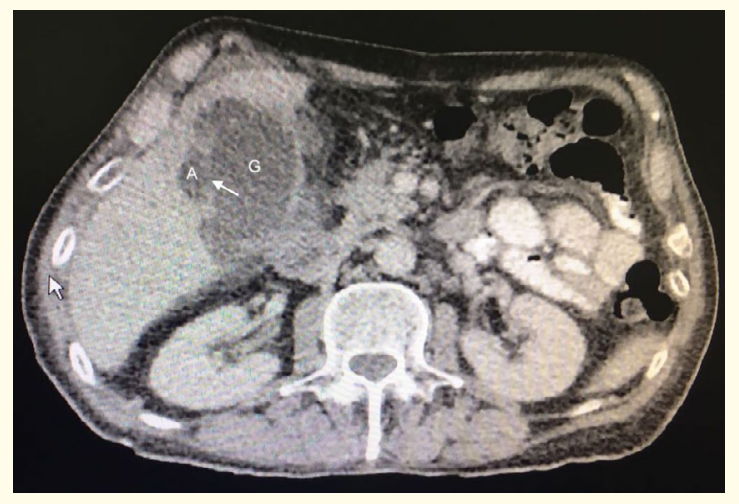

Figure 4: CT shows the sign of the hole (white arrow). Gallbladder (G), abscess (A).

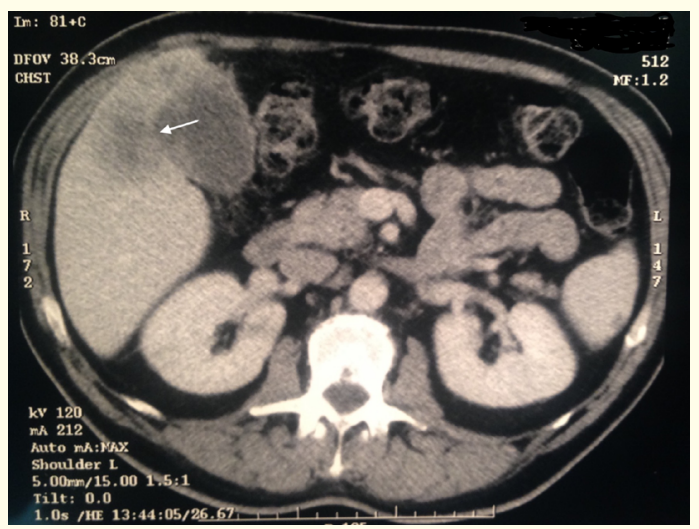

Figure 5: CT shows the sign of the hole (white arrow).

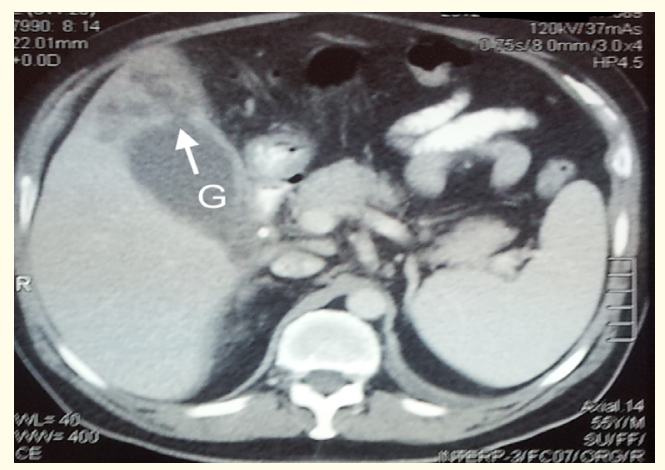

Figure 6: CT shows the sign of the hole (white arrow). Gallbladder (G).

With the ultrasound and the tomography, the diagnosis of liver abscess can be made in the majority of the cases and that leads to the percutaneous treatment (aspiration and drainage) [3,6,13,19,21].

The percutaneous drainage guided by images is nowadays the treatment of choice to liver abscesses [26-29]. This barely invasive procedure that can be done guided by images with minimum morbidity, and the rate of success is up to $90 \%$ [22-25]. With these patients usually a fast resolution of the infectious focus is achieved, allowing the resolution of the biliary pathology deferred, with a much healthier patient, and a very low rate of morbimortality [3,6,13,19,21-29].

The morbidity and mortality of the surgical treatment of liver abscesses has always been high [26-29]. With the upcoming of the barely invasive procedures guided by images, morbimortality has decreased. Nowadays, percutaneous drainage associated to empirical antibiotics represents the treatment of choice. Small abscesses can be treated with percutaneous aspiration, which also allows us to take samples for cultures. Abscesses larger than $5 \mathrm{~cm}$ usually require the placing of a percutaneous drainage catheter [26-29].

The available bibliography suggests that the percutaneous treatment of the liver abscesses is safe and effective, leaving aside the surgical drainage [26-29]. The surgical drainage becomes the treatment of choice when the percutaneous treatment fails, when there is an access difficulty due to anatomic location, or the presence of an associated disease which requires open surgery treatment $[25,28,30,31]$.

The majority of authors express that the liver abscess treatment has to be made by percutaneous drainage initially and deferred Cholecystectomy, and the objective is to take the patients in best shape possible to surgery. About these patients, the treatment is based on percutaneous treatment with abscess drainage and/or percutaneous cholecystostomy. In many cases, given the communication of the abscess with the gallbladder, the use of an only drain- 
age to evacuate the abscess and the gallbladder becomes effective, a situation evidenced in some of our patients [25,30,31].

Most reports suggest the laparotomic approach given the anatomic characteristic of the gallbladder (intrahepatic gallbladder), which associates to high rates of conversion in laparoscopic surgery $[3,6,13,19,21,25,30,31]$.

\section{Conclusion}

- $\quad$ Liver abscesses secondary to acute Cholecystitis are rare but severe.

- Diagnosis is based on clinical suspicion and imageology confirmation, mainly by CT Scan.

- $\quad$ The barely invasive treatment, percutaneous, guided by images, is the treatment of choice. The treatment is based on drainage of the abscess and/or Cholecystostomy. It is an effective treatment, temporizing in gravely ill patients, with a very low rate of morbimortality and in many cases definitive.

\section{Bibliography}

1. Berdirli A., et al. "Factors effecting the complications in the natural history of acute cholecystitis". Hepatogastroenterology 48 (2001): 1275-1278.

2. Stefanidis D., et al. "Gallbladder perforation: Risk factors and outcome". 1 (2006): 204-208.

3. Kochar K., et al. "Intrahepatic perforation of the gall bladder presenting as liver abscess: case report, review of literature and Niemeier's classification". European Journal of Gastroenterology and Hepatology 20 (2008): 240-244.

4. Niemeier OW. "Acute free perforation of the gall bladder". Annuals of Surgery 99 (1934): 922-924.

5. Izadi K., et al. "Gallstone liver abscess secondary to gallbladder perforation”. Radiology Case Reports 4 (2009): 280.

6. Masood MR., et al. "Hepatic abscess secondary to gallbladder perforation: Case report and literature review". SMJ 53 (2008): 60 .

7. Chong VH., et al. "Spontaneous gallbladder perforation, pericholecystic abscess and cholecystoduodenal fistula as the first manifestations of gallstone disease". Hepatobiliary and Pancreatic Diseases International 8 (2009): 212-214.

8. Kimura Y., et al. "Definitions, pathophysiology, and epidemiology of acute cholangitis and cholecystitis: Tokyo Guidelines". Journal of Hepato-Biliary-Pancreatic Sciences 14 (2007): 1526.

9. Menakuru SR., et al. "Current management of gall bladder perforations". ANZ 74 (2004): 843-846.
10. Fletcher AG and Ravdin IS. "Perforation of the gallbladder". The American Journal of Surgery 81 (1951): 178-185.

11. Anderson BB and Nazem A. "Perforations of the gallbladder and cholecystobiliary fistulae: a review of management and a new classification". Journal of the National Medical Association 79 (1987): 393-399.

12. Teefey SA and Wechter DG. "Sonographic evaluation of pericholecystic abscess with intrahepatic extension". Journal of Ultrasound in Medicine 6 (1987): 659-652.

13. Chen JJ., et al. "Gallbladder perforation with intrahepatic abscess formation". Journal of Clinical Ultrasound 18 (1990): 4345.

14. Guy-Grand B., et al. "Liver abscess caused by gallbladder rupture during pyocholecystitis". Sem Hopp 47 (1971): 27372740 .

15. Pratschke E and Berger H. "Perforation of the gall bladder into the liver in gallstone occlusion of the cystic duct". Chirurg 60 (1989): 433-434.

16. Teebken OE., et al. "Chronic cholecystitis simulating gallbladder tumour with liver abscess. Case report". Swiss Surgery 7 (2001): 28-31.

17. Madrazo BL., et al. "Sonographic findings in perforation of the gallbladder". American Journal of Roentgenology 139 (1982): 491-496.

18. Peer A., et al. "Intrahepatic abscess due to gallbladder perforation". Abdom Imaging 20 (1995): 452-455.

19. Bakalakos EA., et al. "Liver abscess secondary to intrahepatic perforation of gallbladder, presenting as a liver mass". American Journal of Gastroenterology 91 (1996): 1644-1646.

20. Fischer MG and Beaton HL. "Unsuspected hepatic abscess associated with biliary tract disease". The American Journal of Surgery 146 (1983): 658-662.

21. Zerman G., et al. "Liver abscess due to acute cholecystitis. Report of five cases”. Chir Italy 55 (2003): 195-198.

22. Donati M., et al. "An athipical presentation of intrahepatic perfrated cholecistitis. A modern indication to open cholecystectomy. Report of a case”. BMC Surgery 14 (2014): 16.

23. Singh K., et al. "Spontaneus intrahepatic type II gallbladder perforation: a rare case of liver abscess-case report". Journal of Clinical and Diagnostic Research 7 (2013): 2,012-2,014

24. Alkofer B., et al. "Are pyogenic liver abscesses still a surgical concern? A Western experience". HBP Surgery (2012): 316,013 . 
25. Hussain T., et al. "Intrahepatic perforation of the galldbladder causing liver abscesses: case studies and literature review of a rare complication". Annals of the Royal College of Surgeons of England 98 (2016): e88-e91.

26. Chou FF., et al. "Single and multiple pyogenic liver abscesses: clinical course, etiology, and results of treatment". World Journal of Surgery 21 (1997): 384-388.

27. Cinat M., et al. "Determinants for successful percutaneous image-guided drainage of intra-abdominal abscess". Archives of Surgery 137 (2002): 845-849.

28. Tan YM., et al. "An appraisal of surgical and percutaneous drainage for pyogenic liver abscesses larger than $5 \mathrm{~cm}$ ". Annuals of Surgery 241 (2005): 485-490.

29. Hope WW., et al. "Optimal treatment of hepatic abscess". American Journal Surgery 74 (2008): 178-82.

30. Costi R., et al. "Synchronous pyogenic liver abscess and acute cholecystitis: how to recognize it and what to do (emergency cholecystostomy followed by delayed laparoscopic cholecystectomy).

31. Kohji Okamoto., et al. "Tokyo Guidelines 2018: flowchart for the management of acute cholecystitis". Journal of HepatoBiliary-Pancreatic Sciences 25 (2018): 55-72.

\section{Volume 2 Issue 9 November 2019}

(C) All rights are reserved by Olivera Pertusso Eduardo., et al. 